Ophthalmologe 2009 · 106:5-5

DOI 10.1007/s00347-008-1894-9

Online publiziert: 14. Dezember 2008

(c) Springer Medizin Verlag 2008

F.G. Holz

Universitäts-Augenklinik Bonn

\title{
Zwei Jahre Erfahrungen mit Editorial Manager
}

\section{Erfolgreiche Umsetzung für "Der Ophthalmologe"}

Version des Redaktionssystems stand bis zu diesem Zeitpunkt noch nicht zur Verfügung.

Heute, nachdem Autoren, Redaktion und Gutachter 2 Jahre mit Editorial Manager $^{\circledast}$ gearbeitet haben, können wir mit Recht sagen, dass die Einführung des Systems für „Der Ophthalmologe“ insgesamt erfolgreich war. Über 200 Beiträge sind seitdem über Editorial Manager ${ }^{\circledast}$ für „Der Ophthalmologe“ eingereicht worden, mehr als je zuvor in vergleichbaren Zeiträumen.

Auch die über 200 für uns tätigen Gutachter der Zeitschrift haben das System, das viele bereits von englischsprachigen Zeitschriften kannten, gerne angenommen, und der Umgang ist routiniert und effizient.

Der gesamte Einreichungs- und Begutachtungsprozess bei „Der Ophthalmologe " konnte mit Editorial Manager ${ }^{\varpi}$ deutlich verkürzt werden, was - verbunden mit dem „Online-First“-Verfahren - zu schnelleren Publikationszeiten geführt hat. Dies dient sowohl den Autoren als auch der raschen Wissensvermittlung.

Es bestehen heute kaum noch Anfragen nach Einreichungs- oder Begutachtungsmöglichkeiten in Papierform, sodass der postalische Versand von Datenträgern und Papierversionen endgültig der Vergangenheit angehört.

Ich freue mich, dass Editorial Manager $^{\oplus}$ von allen Beteiligten so gut akzeptiert wird und danke an dieser Stelle allen Autoren und Gutachtern für ihre stetige, hervorragende Unterstützung und Mitarbeit bei „Der Ophthalmologe“.

Den Leserinnen und Lesern wünsche ich, auch im Jahr 2009 vielfältige Anregungen und Wissenszuwachs bei der Lektüre von „Der Ophthalmologe“!

Ihr

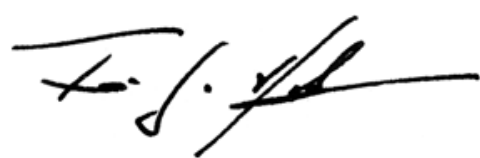

Frank G. Holz

Herausgeber „Der Ophthalmologe“

\section{Korrespondenzadresse}

\section{Prof. Dr. F.G. Holz}

Universitäts-Augenklinik Bonn

Ernst-Abbe-Straße 2, 53127 Bonn

frank.holz@ukb.uni-bonn.de

Interessenkonflikt. Der korrespondierende Autor gibt an, dass kein Interessenkonflikt besteht.

\section{Infobox 1}

Wollen auch Sie ein Manuskript für „Der Ophthalmologe" einreichen?

Reichen Sie Manuskripte für die Rubriken "Übersichten", Originalien", Kasuistiken" und „Bild und Fall“ über das Online-System „Editorial Manager" ein.

Wählen Sie hierzu auf der Zeitschrifthomepage www.DerOphthalmologe.de den Navigationspunkt "Für Autoren" und anschließend "Manuskript online einreichen".

Bei Fragen zur Einreichung wenden Sie sich bitte an:

Frau Dr. Suzan Hunt

Universitäts-Augenklinik Bonn Suzan.Hunt@ukb.uni-bonn.de Tel.: 02244-918491 schrift bei Autoren und Gutachter Akzep$\tan z$ finden würde. Eine deutschsprachige 